Supplementary Information for:

\title{
Planar Double-Epsilon-Near-Zero Cavities for Spontaneous Emission and Purcell Effect Enhancement.
}

\author{
Vincenzo Caligiuri $^{* \dagger}$, Milan Palei ${ }^{\dagger \$}$, Muhammad Imran ${ }^{\dagger \S}$, Liberato Manna ${ }^{\dagger}$ and Roman Krahne ${ }^{* \dagger}$ \\ ${ }^{\dagger}$ Nanochemistry Department, Istituto Italiano di Tecnologia, Via Morego 30, 16163 Genova, Italy \\ ${ }^{\S}$ Dipartimento di Chimica e Chimica Industriale, Università degli Studi di Genova, Via Dodecaneso, \\ 31, 16146 Genova, Italy
}

\section{Contents:}

1. Details on ellipsometric analysis

2. Comparison between Effective Medium Theory and experimental effective dielectric permittivity

3. SEM analysis of NC films

4. Details on the fitting of PL decay traces

5. Emission properties of devices with a $5 \mathrm{~nm}$ thick $\mathrm{Al}_{2} \mathrm{O}_{3}$ spacer layer

Pages S1-S5, Figures S1-S6.

\section{Ellipsometry:}

One of the key strengths of the investigation reported here resides in the experimental demonstration of the epsilon-near-zero (ENZ) nature of the bulk-plasmon-polaritons (BPPs) originating in the plasmonic multilayers. The optical constants of both the metal-insulator-metal (MIM) and metal-insulator-metal insulator-metal (MIMIM) systems have been characterized by using spectroscopic ellipsometry. This analysis consists in the evaluation of the ratio $\varrho=r P / r S$ between the p-polarized and the s-polarized waves reflected from the samples. Starting from this measure, it is possible to evaluate the ellipsometric angles $\Psi$ and $\Delta$ by simply applying the well-known relation $\varrho=\tan (\Psi) \exp (i \Delta)$. Fitting the experimental data for $\Psi$ and $\Delta$ leads to a precise modeling of the optical constants of the analyzed system. Figure S1 shows the fit of $\Psi$ and $\Delta$ for the MIMIM structure. Here it is worth noting that, in order to achieve a correct homogenization of its optical response, the model shown here considers the MIMIM as a single material rather than as a multilayer of known fundamental components with known thickness. Such a technique allows to finely individuate the ENZ wavelengths of the MIMIM structure. 

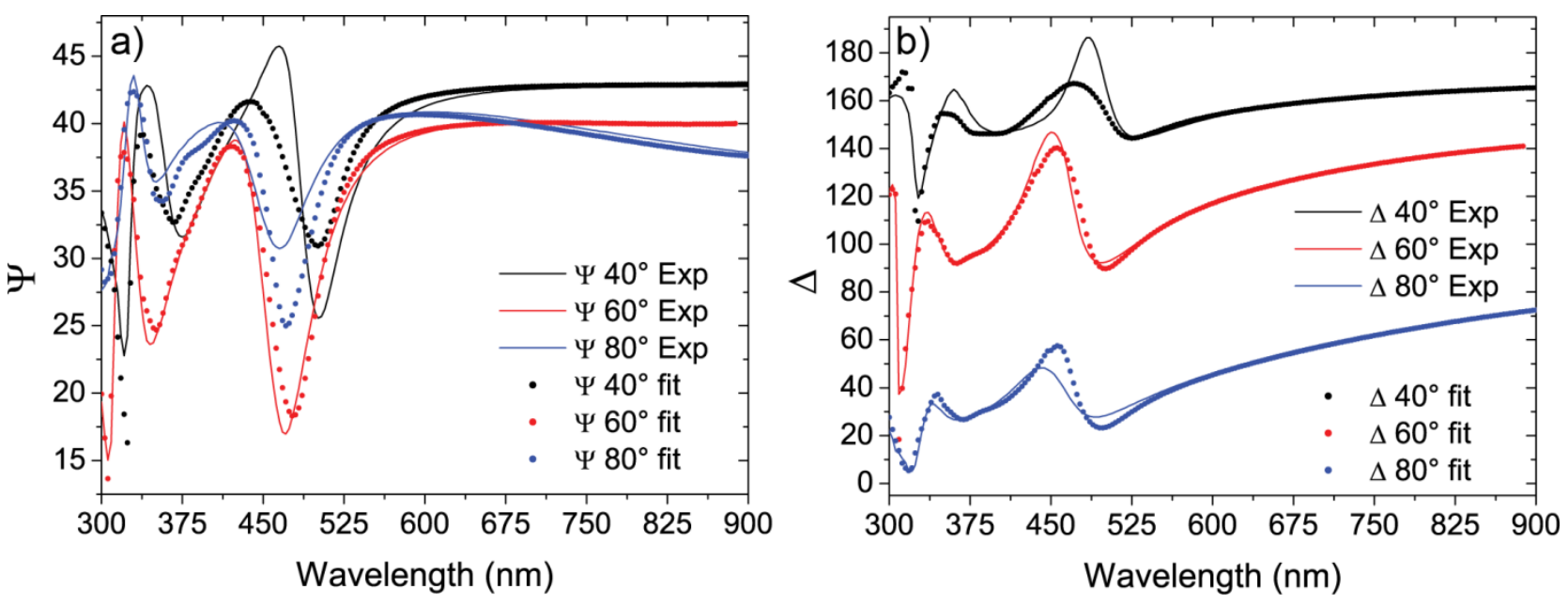

Figure S1. Ellipsometrically measured (a) $\boldsymbol{\Psi}$ and (b) $\Delta$ (solid lines) of a MIMIM structure together with their fits (dotted curves).

The obtained homogenized dielectric permittivity allows an accurate modeling of the optical response of the multilayer, capturing its ENZ features. Figure S2 shows a comparison between the experimentally measured MIMIM reflectance and the Fresnel p-polarized reflectance calculated by replacing the original multilayer with a new substrate whose dielectric permittivity coincide with the ellipsometrically measured one. The very good agreement between experiments and calculations confirms the accuracy of such a homogenization, at the same time validating the ENZ nature of the BPP modes of the MIMIM structure.

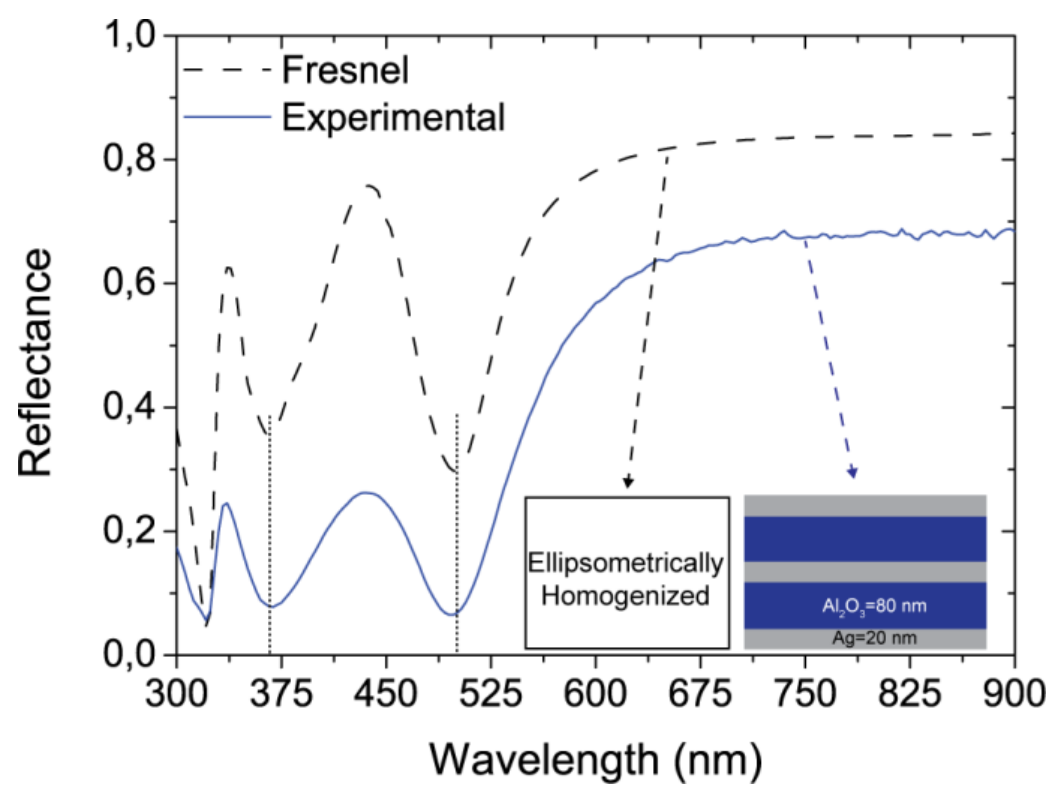

Figure S2. Experimental (solid blue curve) and Fresnel equation reflectance (dashed black curve) calculated by replacing the MIMIM structure with a new substrate possessing the ellipsometrically measured dielectric permittivity. 


\section{Comparison between Effective Medium Theory and experimental effective dielectric permittivity:}

The optical properties of metal/dielectric stacks made of a sufficiently high number of bilayers are usually calculated in the framework of the Effective Medium Theory (EMT). Such an approximation is able to take into account the extreme anisotropic nature of the multilayers, giving at the same time a very good estimation of some of their principal features such as the topological transitions ${ }^{[1]}$. According to the EMT, a metal/dielectric periodic multilayer exhibits two dielectric permittivities, one parallel to the surface of the layers, $\left(\varepsilon_{\|}\right)$and one perpendicular to them $\left(\varepsilon_{\perp}\right)$, whose well known expressions are: ${ }^{[2]}$

$\varepsilon_{\|}=\frac{\varepsilon_{D} t_{D}+\varepsilon_{M} t_{M}}{t_{D}+t_{M}} ;$
$\varepsilon_{\perp}=\frac{\varepsilon_{D} \varepsilon_{M}\left(t_{D}+t_{M}\right)}{\varepsilon_{D} t_{M}+\varepsilon_{M} t_{D}} ;$

However, the resonant propagation regime occurring in stacks made of an exiguous number of metal/dielectric bilayers is completely neglected by the EMT. In order to demonstrate this discrepancy, we report in figure $\mathrm{S} 3$ a comparison between the real part of $\varepsilon_{\|}$and $\varepsilon_{\perp}$ calculated with the equation $\mathrm{S} 1$, together with the experimentally measured effective permittivity by means of ellipsometry:

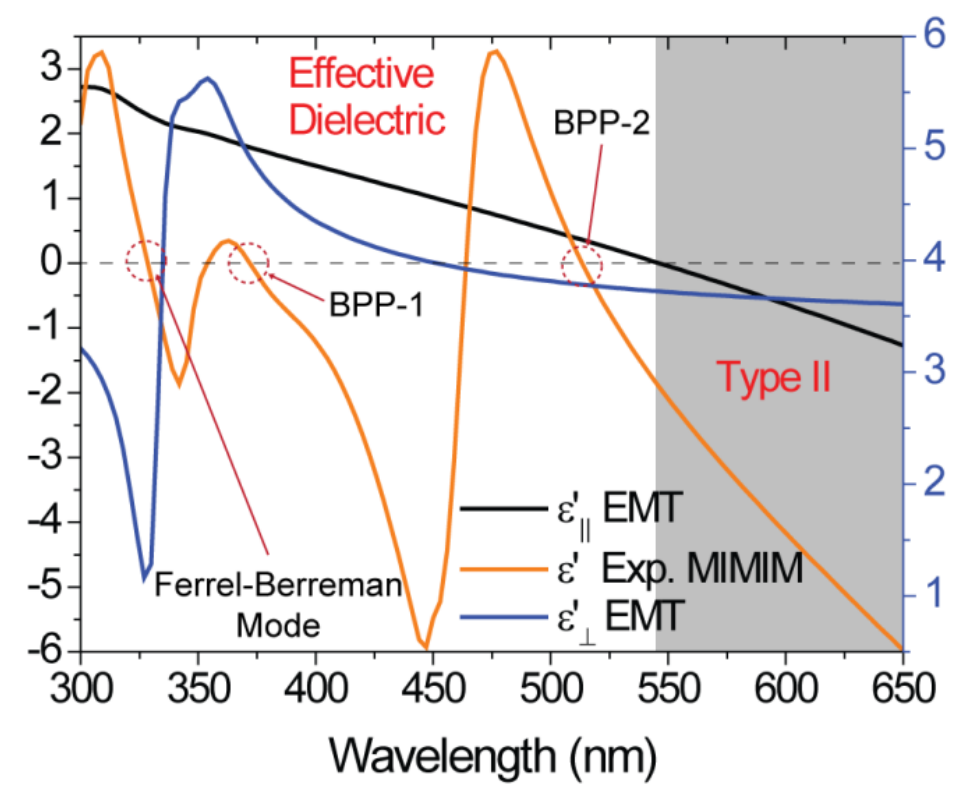

Figure S3: Comparison between the experimental, ellipsometrically measured effective dielectric permittivity and the EMT expressions of $\varepsilon_{\|}$and $\varepsilon_{\perp}$.

As we discuss in the main text, a MIMIM subwavelength structure constitutes a double plasmonic cavity. As such, it is possible to confine two plasmonic modes, corresponding to the so-called BPPs inside the dielectric 
layers that are sandwiched between nanometric metal mirrors. Figure 2 demonstrates that the plasmonic modes correspond to an ENZ response for each cavity that match exactly the confined wavelengths. The orange curve in figure S3 represents the experimental ellipsometrically measured dielectric permittivity of the MIMIM structure. The occurrence of the ENZ condition at both the two BPPs, as well as at the FerrelBerreman mode (around 327nm) is well evident as a zero crossing (see red dotted circles) of the dielectric permittivity curve. The effective permittivities (red and black curves) calculated by EMT completely neglect these features, and therefore are unable to describe the propagation in the two epsilon-near-zero regimes as well as in the Ferrel-Berreman mode.

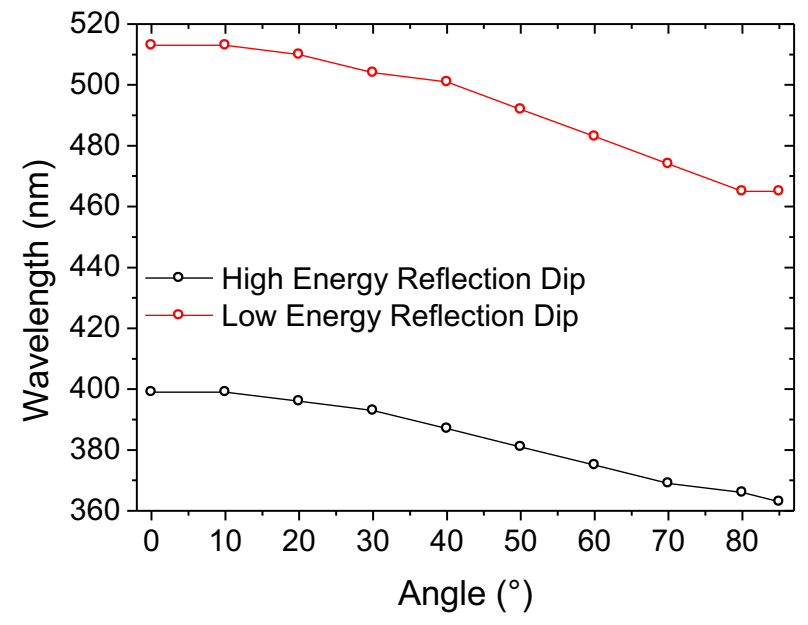

Figure S4. Dependence of the Bulk-Plasmon-Polariton modes on the angle of incidence of a plane wave modeled by Scattering Matrix Simulations.

\section{SEM analysis of NC films:}

Scanning Electron Microscope (SEM) images recorded of the $\mathrm{CsPbBr}_{3}$ films spin-coated on (a) bare Al2O3, (b) MIM, and (c) MIMIM structures are reported in figure S5 and reveal the homogeneity of all the three films.
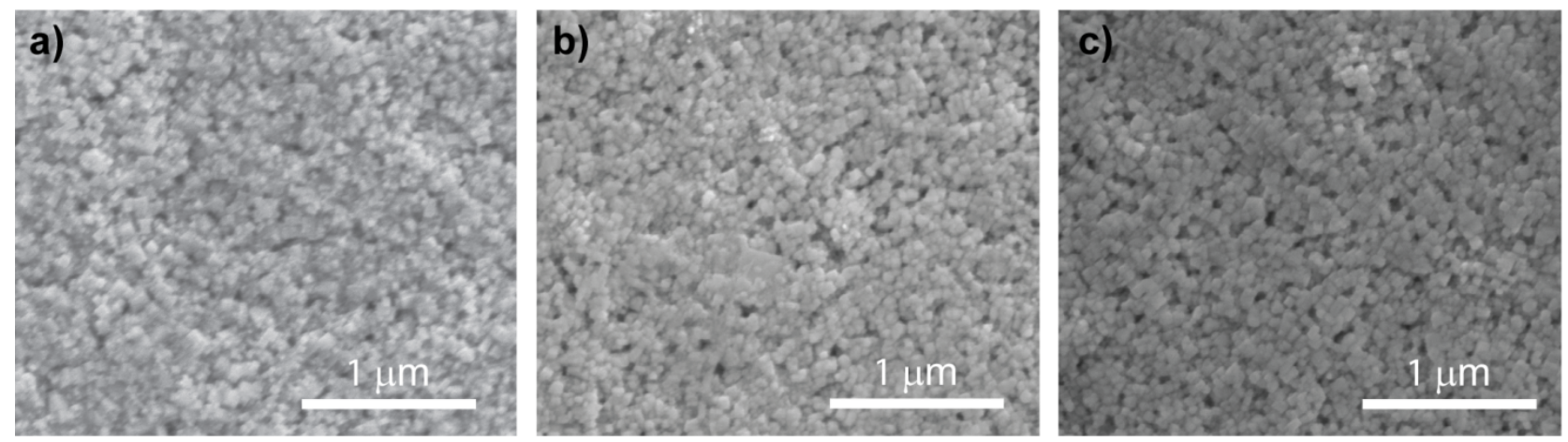

Figure S5. SEM analysis demonstrating the homogeneity of the NC films deposited on (a) bare alumina substrate, (b) MIM and, (c) MIMIM samples. 


\section{Fitting of PL decay:}

The decay traces of the PL were fitted by a classic three-exponential function:

$$
A_{1} \cdot e^{\left(\frac{t}{\tau_{1}}\right)}+A_{2} \cdot e^{\left(\frac{t}{\tau_{2}}\right)}+A_{3} \cdot e^{\left(\frac{t}{\tau_{3}}\right)}
$$

The average life times have been calculated by using the intensity-weighted relation:

$$
\bar{\tau}=\sum_{i} f_{i} \tau_{i}, \text { where } f_{i}=\frac{A_{i} \tau_{i}}{\sum_{j} A_{j} \tau_{j}} \text {. Therefore } \tau_{A V G} \text { is: } \bar{\tau}=\frac{A_{1} \tau_{1}^{2}+A_{2} \tau_{2}^{2}+A_{3} \tau_{3}^{2}}{A_{1} \tau_{1}+A_{2} \tau_{2}+A_{3} \tau_{3}} \text {. }
$$

5. Emission properties of devices with a thin, $5 \mathrm{~nm}$ thick, $\mathrm{Al}_{2} \mathrm{O}_{3}$ spacer layer between the perovskite nanocrystal film and the metal/dielectric multilayers.
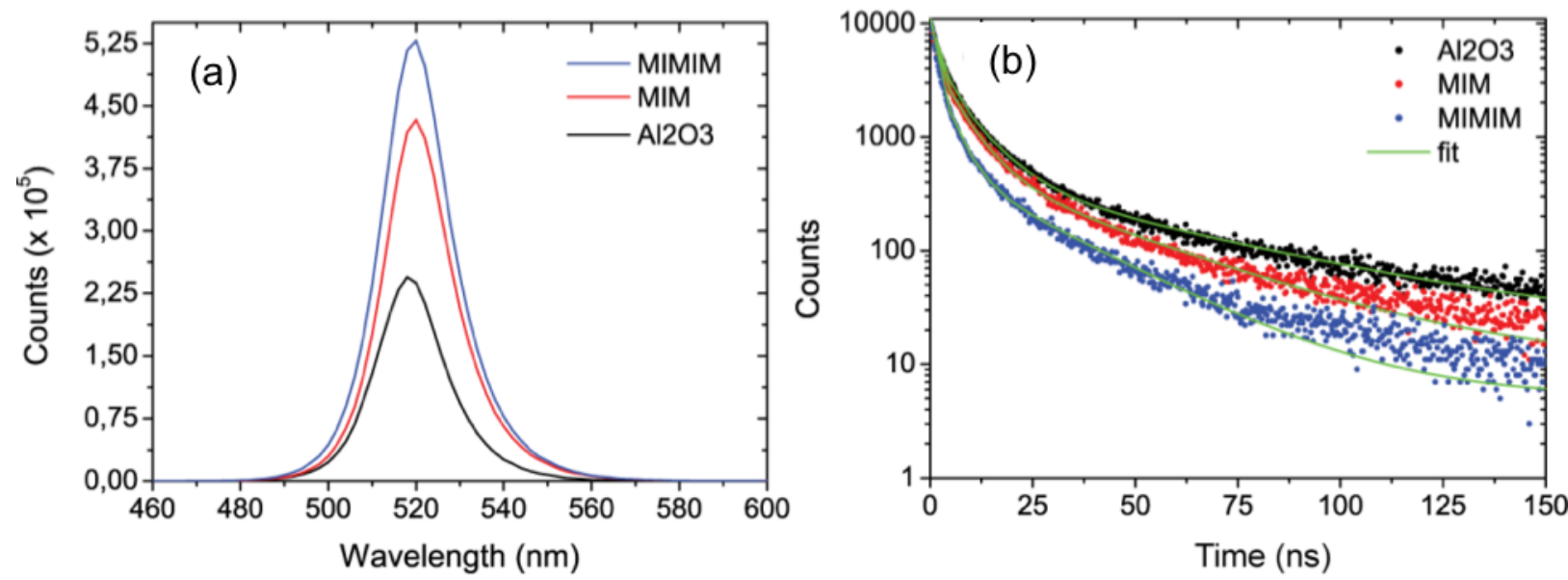

Figure S6. Spontaneous emission (a) and decay traces (b) of Cs $\mathrm{PbBr}_{3}$ nanocubes deposited on a bare $\mathrm{Al}_{2} \mathrm{O}_{3}$ substrate (black), a MIM (red) and MIMIM (blue) structure. For the MIM and MIMIM structures the top alumina spacer layer had a thickness of $5 \mathrm{~nm}$.

The Time Correlated Single Photon Counting (TCSPC) measurements displayed in Figure S6b reveal the spontaneous decay rate, and the fitting results with a three-exponential decay are reported in Table S1.

Table S1. Decay lifetimes from structures with a $5 \mathrm{~nm}$ thick spacer layer obtained by fitting the decay traces with a three-exponential function for the three systems consisting in simple $\mathrm{Al}_{2} \mathrm{O}_{3}, \mathrm{MIM}$ and complete MIMIM.

\begin{tabular}{|c|c|c|c|}
\hline & $\boldsymbol{\tau}_{\mathbf{1}}(\mathbf{n s})$ & $\boldsymbol{\tau}_{\mathbf{2}}(\mathbf{n s})$ & $\boldsymbol{\tau}_{\mathbf{3}}(\mathbf{n s})$ \\
\hline $\mathbf{A l}_{\mathbf{2}} \mathbf{O}_{\mathbf{3}}$ & 2.21 & 8.08 & 48 \\
\hline $\mathbf{M I M}$ & 1.41 & 6.33 & 32.76 \\
\hline MIMIM & 1.15 & 4.04 & 23.41 \\
\hline
\end{tabular}

